\title{
Atherosclerosis-Preventing Activity of Lactic Acid Bacteria-Fermented Milk-Soymilk Supplemented with Momordica charantia
}

\author{
Tsung-Yu Tsai, Li-Han Chu, Chun-Lin Lee, and Tzu-Ming Pan* \\ Institute of Microbiology and Biochemistry, National Taiwan University, 1, Section 4, Roosevelt \\ Road, Taipei, Taiwan, Republic of China
}

\begin{abstract}
In this study, the milk-soymilk and milk-soymilk supplemented with Momordica charantia, a common oriental vegetable possessing medicinal activities, were fermented by lactic bacteria. The objective of this study was to investigate the effects of milk-soymilk and fermented milk-soymilk with or without M. charantia on atherosclerosis in hyperlipidemic hamsters. Fermented $25 \%$ milk and $75 \%$ soymilk combinations, supplemented with $1 \% M$. charantia solution, can improve the acceptability of the fermented beverage. A total of 72 male Golden Syrian hamsters were divided into 9 groups $(n=$ 8/group), and experimental diets were provided with a normal diet for the normal group and a highcholesterol diet for others. The milk-soymilk and fermented milk-soymilk with or without $M$. charantia were administrated for 8 weeks. The milk-soymilk and fermented milk-soymilk with and without $M$. charantia were able to significantly decrease $(p<0.05)$ the serum cholesterol and the atherosclerotic plaque in aorta based on the comparison to the high-cholesterol diet $(\mathrm{H})$ group. The groups on fermented milk-soymilk by Lactobacillus plantarum NTU 102 with or without $M$. charantia could significantly decrease $(p<0.05)$ the ratio of low-density lipoprotein cholesterol (LDL-C) to highdensity lipoprotein cholesterol (HDL-C). The femented milk-soymilk by Lactobacillus paracasei subsp. paracasei NTU 101 supplemented with $M$. charantia had an anti-atherosclerotic activity by increasing superoxide dismutase (SOD) and total antioxidant status (TAS) activity of the blood and relieving the degree of thiobarbituric acid reactive substances (TBARS) compared to the other treatments. It is concluded that the milk-soymilk and the fermented milk-soymilk supplemented with or without $M$. charantia by L. paracasei subsp. paracasei NTU 101 are effective in preventing and retarding the hyperlipidemia-induced oxidative stress and atherosclerosis.
\end{abstract}

KEYWORDS: Atherosclerosis; bitter melon; hyperlipidemia; lactic acid bacteria; milk-soymilk; Momordica charantia

\section{INTRODUCTION}

Soybeans provide high-quality proteins, fats, and carbohydrates and contain no cholesterol or lactose. They are a plentiful and inexpensive nutritive source for lactose-intolerant consumers, vegetarians, and milk-allergy patients (1). Isoflavones are found abundantly in soybeans, with a structure homology to human estrogens (2). Several studies suggest isoflavones have some useful biological effects for hormone-dependent diseases, such as the prevention of breast, prostate, and colon cancers, cardiovascular disease, osteoporosis, postmenopausal symptoms, and anti-obesity activities (3). The biological activities of soy isoflavones are different depending upon their chemical forms (4). The parent isoflavones are the aglycone structures of daidzein, genistein, and glycetein, which are conjugated to form

* To whom correspondence should be addressed. Telephone: $+886-$ 2-3366-4519 ext. 10. Fax: +886-2-2362-7044. E-mail: tmpan@ ntu.edu.tw. malonyl-, acetyl-, and $\beta$-glucoside configurations. In general, isoflavones in soybeans and unfermented soy foods exist mainly as glucoside forms and rarely as aglycone forms; in addition, the bioavailability of aglycones and glucosides of soy isoflavones is controversial. However, some studies indicated that the bioavailability of these glucosides required initial hydrolysis of the sugar moiety by intestinal $\beta$-glucosidases, which increase absorption of isoflavone aglycones in healthy adults. Biotransformation and the production of metabolites of isoflavones in the intestinal tract are highly dependent upon the nature of intestinal microflora and fermentation products; furthermore, lactic acid bacteria (LAB) can influence the absorption or metabolism of isoflavones $(5,6)$.

LAB are important members of the normal intestinal microflora and are reported to exert beneficial effects, including the inhibition of the growth of potential pathogens, reduction of serum cholesterol, and the modulation of the immunity system $(7,8)$. LAB are widely used in the food industry to 
produce a variety fermentation foods. They are also used in the production of industrial chemicals, biological products, and food biopreservatives. More recently, LAB are strong candidates for the development of oral delivery vehicles for digestive enzymes and the development of mucosal vaccine antigens $(9,10)$. In addition, they have been found to possess $\beta$-glucosidases, which are responsible for an increase in isoflavone aglycone components in fermented soymilk. Therefore, soymilk fermented by LAB may lead to a combination of benefits as probiotics as well as that from the transformation of isoflavone glucosides to bioactive isoflavone aglycones (11).

Momordica charantia L. (bitter melon), a common oriental vegetable, possesses medicinal activities including a reduction of blood glucose and lipids in both normal and diabetic animals. In addition, it may protect $\beta$ cells, enhance insulin sensitivity, reduce oxidative stress, and possess immunoadjuvant activity (12). Bitter melon also exhibited a marked reduction in the hepatic total cholesterol and triglyceride levels in both the presence and absence of dietary cholesterol (13). In this study, the milk-soymilk and milk-soymilk supplemented with $M$. charantia were fermented by LAB. The objective of this study was to investigate the effects of milk-soymilk and the fermented milk-soymilk containing $M$. charantia on atherosclerosis in hyperlipidemic hamsters.

\section{MATERIALS AND METHODS}

Chemicals and Media. Thiobarbituric acid (TBA), malondialdehyde (MDA), and dimethyl sulfoxide (DMSO) were purchased from Sigma Chemical Co. (St. Louis, MO). Lactobacilli MRS broth and Bactoagar were purchased from Difco Co. (Detroit, MI). Soybeans [Glycine $\max ($ L.) Merrill] and the fruits of M. charantia were purchased from supermarkets (Taipei, Taiwan).

Preparation of Soymilk and Fermented Soymilk by LAB. The preparation of soymilk was carried out with the substrate of nongenetically modified soybeans and skim milk powder purchased from a local supermarket in Taiwan. Briefly, soybeans were soaked in deionized water for $8 \mathrm{~h}$ at $25^{\circ} \mathrm{C}$. The swollen beans were ground into a homogenate with water of 8 times soybean dry weight using a blender, and the homogenate was then filtered through a defatted cotton sheet. Milk was $8 \%(\mathrm{w} / \mathrm{v})$ reconstituted skim milk powder. The milk and soymilk were heat-treated individually in water baths at $90{ }^{\circ} \mathrm{C}$ for $1 \mathrm{~h}$. After heat treatment and cooling to room temperature, milk and soymilk were mixed at a proportion of 25:75 (v/v), respectively. The fruits of $M$. charantia were purchased from a local supermarket in Taipei, Taiwan, mixed with 2 times its weight in distilled water, and blended for $3 \mathrm{~min}$. The resultant slurry was filtered through a sieve and then heated in a water bath at $90{ }^{\circ} \mathrm{C}$ for $1 \mathrm{~h}$. After heat treatment and cooling to room temperature, $M$. charantia juice was added to the milk-soymilk mixtures at the rate of $5,10,15$, and $20 \%(\mathrm{v} / \mathrm{v})$, respectively. Lactobacillus paracasei subsp. paracasei NTU 101 and Lactobacillus plantarum NTU 102, which were isolated from infant feces and fermented vegetables in Taiwan, were inoculated in milk-soymilk (14). The culture strains, $L$. paracasei subsp. paracasei NTU 101 and $L$. plantarum NTU 102, were enriched in lactobacilli MRS broth at 37 ${ }^{\circ} \mathrm{C}$ for $48 \mathrm{~h}$ and inoculated $1 \%(\mathrm{v} / \mathrm{v})$ to milk-soymilk, respectively. All cultured milk-soymilk were incubated in flasks at $37^{\circ} \mathrm{C}$ for $72 \mathrm{~h}$. At the end of preparation, all samples were dried by a freeze-dryer (SDF-25, Chang Jung Business Co., Ltd., Feng-Jen, Taiwan) for analyzing and feeding the hamsters. The glycoside and aglycone isoflavones were analyzed by high-performance liquid chromatography (HPLC) (Jasco Co., Tokyo, Japan) acording to Lin et al. (15).

Sensory Evaluation. The milk-soymilk supplemented with 5, 10, $15,20 \% M$. charantia solution fermented by $L$. paracasei subsp. paracasei NTU 101 and L. plantarum NTU 102 were evaluated for their organoleptic properties. Sensory evaluation was carried out after $24 \mathrm{~h}$ of storage at $4{ }^{\circ} \mathrm{C}$ of the fermented products. Samples served (about $4{ }^{\circ} \mathrm{C}$ ) were scored on a $1-9$ hedonic scale ( 9 was considered excellent; 5 was considered acceptable; and 1 was considered extremely
Table 1. Composition of the Experimental Diets ${ }^{a}$ ( $/ / k g$ Diet)

\begin{tabular}{lcc}
\hline \multicolumn{1}{c}{ composition } & normal diet $^{b}$ & high-cholesterol diet \\
\hline casein & 200 & 139 \\
corn starch & 650 & 680 \\
cellulose & 50 & 50 \\
soybean oil & 50 & 80 \\
mineral & 35 & 35 \\
vitamin & 10 & 10 \\
L-cysteine & 3 & 1 \\
choline bitartrate & 2 & 2 \\
cholesterol & & 2 \\
cholic acid & & 1
\end{tabular}

${ }^{a}$ On the basis of the AIN-76 formula. ${ }^{b}$ The daily diet of the control group.

poor). A panel of 60 untrained students evaluated each sample. The characteristics evaluated included color, flavor, taste, texture, and overall acceptability. A fermented milk-soymilk beverage without adding $M$. charantia juice was used as the control. Duncan's multiple range tests were used to determine significant difference.

Animals and Diets. A total of 72 male Golden Syrian hamsters weighing 100-120 g were housed in individual plastic cages and subjected to a $12 \mathrm{~h}$ light/dark cycle, with a maintained relative humidity of $60 \%$ and a temperature at $25{ }^{\circ} \mathrm{C}$. The animals were given free access to regular rodent feed and water for 4 weeks to adapt to the new environment. Hamsters were weighed and randomly assigned into 9 groups of 8 animals each before the animal experiment.

Dose and Grouping. The dose of milk-soymilk was calculated in accordance with Boyd's formula of body surface area as recommended by the Food and Drug Administration (FDA) (16). The composition of expirimental diets were shown in Table 1. The daily dietary dose of commercial soymilk product is usually recommended and used at $400 \mathrm{~mL}$, which consisted of $15 \mathrm{~g}$ of total proteins for the adult. In a previous study, $15 \mathrm{~g}$ of total proteins in soymilk was used as a reference dosage for an adult as hypolipilidemic effects (17). Probucol as the positive hypolipilidemic drug was also recommended at $1 \mathrm{~g} /$ day for an adult (16). After the prebreeding stage for 4 weeks, all test samples were suspended in $1 \mathrm{~mL}$ of water and orally administrated to the hamsters using a stomach tube for 8 weeks. The food intake was recorded daily, and animals were weighed weekly. Experimental diets were provided in accordance with AIN-76 diet formulation, with modification. The $\mathrm{N}$ group was fed a normal diet via AIN-76 formulation, and the $\mathrm{H}$ group was given a high-cholesterol diet that contained $0.2 \%$ cholesterol (14). HS, HSF-101, and HSF-102 groups were fed the high-cholesterol diet and orally given milk-soymilk and fermented milk-soymilk by L. paracasei subsp. paracasei NTU 101 and L. plantarum NTU 102 (4.5 g/kg BW per day including $15 \mathrm{~g}$ of total proteins), respectively. HM, HMF-101, and HMF-102 groups were also fed the high-cholesterol diet and orally given milk-soymilk and fermented milk-soymilk by L. paracasei subsp. paracasei NTU 101 and L. plantarum NTU 102 supplemented with $1 \%$ (v/v) M. charantia (5.5 g/kg BW per day including $15 \mathrm{~g}$ of total proteins), respectively. In addition, the HP group, a positive control group, was fed the highcholesterol diet and given orally 1 -fold dose of probucol $(100 \mathrm{mg} / \mathrm{kg}$ BW per day).

At $24 \mathrm{~h}$ before sacrifice, all food was removed. Animals were anesthetized by carbon dioxide inhalation and sacrificed. Whole blood were collected, and plasma samples were prepared by sodium citrate and then stored at $-80{ }^{\circ} \mathrm{C}$. Liver tissue was cleaved and rinsed frequently with steriled $0.8 \%$ sodium chloride solution to eliminat blood. Liver tissue was ground in ice-cold steriled phosphate buffer saline (PBS) and then centrifuged ( $8000 \mathrm{~g}$ for $15 \mathrm{~min}$ ). The supernatant was collected and stored at $-80{ }^{\circ} \mathrm{C}$ for the assay of superoxide dismutase (SOD) activity and thiobarbituric acid reactive substances (TBARS). The other liver tissue was immersed in the liquid nitrogen and then stored at $-80{ }^{\circ} \mathrm{C}$. The experiment was reviewed and approved by the Animal Care and Research Ethics Committee of the National Taiwan University.

Serum and Liver Lipid Analysis. Serum total cholesterol (TC), triglycerol (TG), and high-density lipoprotein cholesterol (HDL-C) levels were measured in triplicate using commercial enzymatic kits. 
Table 2. Average Scores for the Sensory Properties of Different Fermented M. charantia Milk-Soymilks

\begin{tabular}{|c|c|c|c|c|c|}
\hline & color & flavor & taste & texture & overall acceptability \\
\hline control $^{a}$ & $5 b$ & $5 a$ & $5 \mathrm{a}$ & $5 b$ & $5 \mathrm{bc}$ \\
\hline $1 \%$ & $5.95 \pm 1.33 \mathrm{a}$ & $4.97 \pm 1.46 a b$ & $5.27 \pm 1.40 \mathrm{a}$ & $5.35 \pm 1.28 \mathrm{a}$ & $5.56 \pm 1.53 \mathrm{a}$ \\
\hline $5 \%$ & $4.60 \pm 1.60 b$ & $4.62 \pm 1.25 b$ & $4.57 \pm 1.18 b$ & $4.38 \pm 1.42 c$ & $4.45 \pm 1.15 c$ \\
\hline $10 \%$ & $4.83 \pm 1.43 b$ & $4.52 \pm 1.43 b$ & $4.55 \pm 1.43 b$ & $5.05 \pm 1.46 a b$ & $4.63 \pm 1.57 c$ \\
\hline $20 \%$ & $3.52 \pm 1.71 c$ & $3.28 \pm 1.46 c$ & $2.95 \pm 1.41 \mathrm{c}$ & $3.83 \pm 1.32 \mathrm{~d}$ & $3.02 \pm 1.31 \mathrm{~d}$ \\
\hline
\end{tabular}

\footnotetext{
${ }^{a}$ The control is milk-soymilk without $M$. charantia solution; $1,5,10$, and $20 \%$ is milk-soymilk with $1,5,10$, and $20 \%$. charantia solution, respectively. Data are
} presented as mean $\pm \mathrm{SD}(n=60)$, and numbers with a different letter were significantly different $(p<0.05)$ from each other.

These kits were as follow: TC assay kit (CH 200, Randox Laboratories Ltd., Antrim, U.K.), TG assay kit (TR-210, Randox Laboratories Ltd.), and HDL-C assay kit (CH-203, Randox Laboratories Ltd.). Serum lowdensity lipoprotein cholesterol (LDL-C) levels were acquired via the follow calculation $(14,16)$, LDL-C $(\mathrm{mg} / \mathrm{dL})=\mathrm{TC}-\mathrm{TG} / 5-\mathrm{HDL}-\mathrm{C}$.

Liver tissue $(0.5 \mathrm{~g})$ was ground in $10 \mathrm{~mL}$ of ice-cold Folch solution [chloroform/methanol $=2: 1(\mathrm{v} / \mathrm{v})]$ and incubated for $30 \mathrm{~min}$ at room temperature. The aqueous layer was aspirated and discarded, and the fixed volume of the organic layer was then evaporated to dryness. The dried lipid layer were dissolved with an equal volume of DMSO and then used to determine the TC and TG levels using commercial enzymatic kits.

Determination of TBARS Content, Total Antioxidant Status (TAS), SOD Activity, and Catalase Activity. TBARS assay is the accepted method for the determination method for in vivo lipid peroxidation (18). According to the procedure of a previous study, TBARS levels of serum and liver were determined by the method of thiobarbituric acid (TBA) colorimetric analysis and the optical density (OD) value was measured at $532 \mathrm{~nm}$. The SOD activities of homogenated liver and erythrocyte from whole blood were examined using the commercial kit (Ransod, Randox Laboratories Ltd.). Determination of the TAS of plasma was carried out using the commercial kit (TASNX2332, Randox Laboratories Ltd.). The assay principle is that metmyoglobin reacts with $\mathrm{H}_{2} \mathrm{O}_{2}$ to form the radical species, ferrylmyoglobin. A chromogen [2,2'-azino-di(ethylbenzthiazoline sulfonate) (ABTS)] is incubated with the ferrylmyoglobin to produce the radical cation species ABTS. This has a relatively stable blue-green color, which is measured at $600 \mathrm{~nm}$. Antioxidants cause suppression of this color production to a degree that is proportional to their concentration (19).

Stain of Aortic Plaque in Artery. The thoracic aorta was cut open longitudinally along the anterior side, and the lipid-rich lesions on the surface of the aorta were stained with $2 \%$ Sudan IV and then successively washed with a gradient concentration of methanol (100, $90,80,70$, and $60 \%$ ) and PBS. The whole surface area of the thoracic aorta was stained by Sudan IV and photographed using a digital camera. The aortic surface area and its stained plaque area (red) were selected and quantitated by the Posterize program of Photoshop 7.0 software (Adobe Systems Incorporated, San Jose, CA). The selected pixels of the plaque area and whole aorta were used to calculate the percent area of the aortic plaque, as follow:

aortic plaque percent $(\%)=$

(pixel of stained plaque area/pixel of whole aorta) $\times 100 \%$

Statistics. Data are expressed as the mean \pm standard deviation (SD). The statistical significance among the groups was determined by oneway analysis of variance (ANOVA) followed by Duncan's multiple range tests.

\section{RESULTS}

Sensory Evaluation. The results of sensory evaluation were shown in Table 2. The mean scores of color of the fermented beverage supplemented with $1 \% \mathrm{M}$. charantia solution were higher than other samples and were significantly different from the control and fermented beverage that were supplemented with $20 \%$ M. charantia solution. Supplementation of $1 \%$ M. charantia solution can improve the color of the control. Although the flavor and taste of $1 \%$ M. charantia solution were not

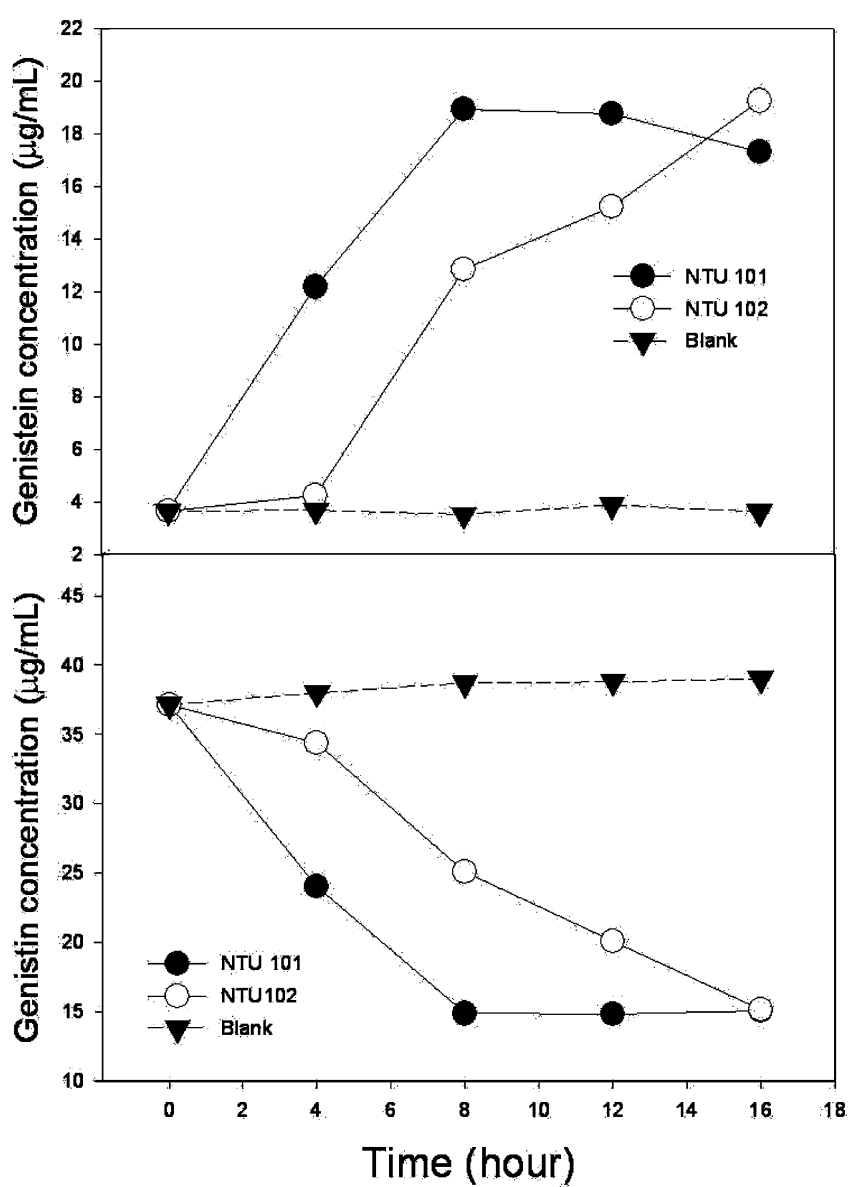

Figure 1. Concentrations of genistin (bottom) and genistein (top) in Lactobacillus-fermented milk-soymilk in the course of $16 \mathrm{~h}$ of fermentation. NTU 101, milk-soymilk fermented by L. Paracasei subsp. paracasei NTU 101 at $37^{\circ} \mathrm{C}$; NTU 102 , milk-soymilk fermented by L. plantarum NTU 102 at $37^{\circ} \mathrm{C}$; Blank, milk-soymilk at $37^{\circ} \mathrm{C}$ without fermentation by LAB.

significantly different from the control, they were distinguishly different from all other samples. The mean scores of the texture of fermented beverages supplemented with $10 \% \mathrm{M}$. charantia solution were significantly higher than 5 and $20 \%$ M. charantia solutions. In overall acceptability, the fermented beverage supplemented with $1 \% M$. charantia solution had the highest mean scores and was significantly different from all other samples. As shown in the results, beverages supplemented with $1 \% M$. charantia solution can improve the acceptability of fermented $25 \%$ milk and $75 \%$ soymilk combinations.

Glycoside and Aglycone Isoflavones in Milk-Soymilk and Fermented Milk-Soymilk. In this study, glycoside isoflavone, genistin, and aglycone isoflavone, genistein, were analyzed at the period of lactic acid fermentation, which was shown in Figure 1. As the result, genistein increased 5-fold (from 3.8 to 
Table 3. Effect of Milk-Soymilk and Fermented Milk-Soymilk on Serum and Liver TC and Serum TG Levels in Hyperlipidemic Hamsters ${ }^{a}$

\begin{tabular}{lccc}
\hline group & $\begin{array}{c}\text { serum cholesterol } \\
(\mathrm{mg} / \mathrm{dL})\end{array}$ & $\begin{array}{c}\text { liver cholesterol } \\
(\mathrm{mg} / \mathrm{dL})\end{array}$ & $\begin{array}{c}\text { serum triglyceride } \\
(\mathrm{mg} / \mathrm{dL})\end{array}$ \\
\hline $\mathrm{N}$ & $89.1 \pm 12.9 \mathrm{a}$ & $15.9 \pm 4.9 \mathrm{a}$ & $79.2 \pm 22.3 \mathrm{a}$ \\
$\mathrm{H}$ & $160.8 \pm 19.9 \mathrm{~d}$ & $34.2 \pm 7.0 \mathrm{c}$ & $128.0 \pm 24.8 \mathrm{~b}$ \\
$\mathrm{HP}$ & $109.6 \pm 16.4 \mathrm{ab}$ & $26.1 \pm 7.1 \mathrm{~b}$ & $98.2 \pm 32.8 \mathrm{ab}$ \\
$\mathrm{HS}$ & $128.0 \pm 29.3 \mathrm{bc}$ & $26.3 \pm 9.9 \mathrm{~b}$ & $106.1 \pm 41.7 \mathrm{ab}$ \\
$\mathrm{HSF}-101$ & $138.0 \pm 27.1 \mathrm{c}$ & $15.3 \pm 4.0 \mathrm{a}$ & $117.2 \pm 26.9 \mathrm{~b}$ \\
HSF-102 & $132.1 \pm 12.3 \mathrm{bc}$ & $17.9 \pm 3.6 \mathrm{ab}$ & $114.1 \pm 33.2 \mathrm{ab}$ \\
HM & $133.7 \pm 22.3 \mathrm{c}$ & $19.9 \pm 4.6 \mathrm{ab}$ & $105.8 \pm 36.1 \mathrm{ab}$ \\
HMF-101 & $124.5 \pm 16.8 \mathrm{bc}$ & $23.6 \pm 4.0 \mathrm{ab}$ & $112.6 \pm 28.4 \mathrm{ab}$ \\
HMF102 & $137.0 \pm 24.2 \mathrm{c}$ & $20.2 \pm 6.9 \mathrm{ab}$ & $125.5 \pm 26.7 \mathrm{~b}$ \\
& & &
\end{tabular}

${ }^{a} \mathrm{~N}$, normal diet $(0 \%$ cholesterol); $\mathrm{H}$, high-cholesterol diet, $\mathrm{HP}$, probucol and high-cholesterol diet; HS, milk-soymilk and high-cholesterol diet; HSF-101, milk-soymilk fermented by $L$. paracasei subsp. paracasei NTU 101 and highcholesterol diet; HSF-102, milk-soymilk fermented by L. plantarum NTU 102 and high-cholesterol diet; HM, milk-soymilk supplement with M. charantia and highcholesterol diet; HMF-101, milk-soymilk supplement with M. charantia fermented by L. paracasei subsp. paracasei NTU 101 and high-cholesterol diet; HMF-102, milk-soymilk supplement with M. charantia fermented by L. plantarum NTU 102 and high-cholesterol diet. Data are presented as mean $\pm \operatorname{SD}(n=8)$, and numbers with a different letter were significantly different $(p<0.05)$ from each other.

$19.5 \mu \mathrm{g} / \mathrm{mL}$ ) at $16 \mathrm{~h}$ by fermenting with L. paracasei. subsp. paracasei NTU 101 and L. plantarum NTU 102 and genistin decreased at the period of fermentation.

Changes in Body Weight and Daily Intake. The results indicate that the body weight and daily intake of the hamsters increased normally and had no difference among the various groups during the period of the practical experiment (data not shown). In addition, the externals and health of all experimental animals had a normal expression.

Hypolipidemic Effect in Serum and Liver. As shown in Table 3, a high-cholesterol diet increased the serum TC level of the $\mathrm{H}$ group by $80.5 \%(p<0.05)$, as compared to the $\mathrm{N}$ group. The other groups (HP, HS, HSF-101, and HSF-102) treated with (1) probucol, (2) milk-soymilk, and (3) fermented milk-soymilk had significant differences with the $\mathrm{H}$ group ( $p$ $<0.05$ ) on the cholesterol levels. The HM, HMF-101, and HMF-102 groups, which were supplemented with $M$. charantia, were also significantly different from the $\mathrm{H}$ group $(p<0.05)$, but no significant hypocholesterolemic effects were found with other treated groups.

The $\mathrm{H}$ group fed with a high-cholesterol diet had a higher serum TG level by $61.6 \%$ than the $\mathrm{N}$ group $(p<0.05)$. All experimental groups had less value but no significant difference with the $\mathrm{H}$ group $(p<0.05)$. Therefore, the hypotriglyceridemic effect of milk-soymilk and fermented milk-soymilk with or without $M$. charantia did not reduce the symptoms of hyperlipidemic hamsters.

The effect of milk-soymilk on lowering TC levels of liver was shown in Table 3. As expected, hamsters treated with a high-cholesterol diet for 8 weeks showed a remarkable increase in liver TC levels, as compared to the $\mathrm{N}$ group ( $p<0.05)$. All treated groups decrease liver TC levels significantly $(p<0.05)$, which were compared to the $\mathrm{H}$ group. However, the significant hypotriglyceridemic effect was not expressed in all treated groups $(p>0.05)$ (data not shown).

Changes in HDL-C, LDL-C, and LDL-C/HDL-C. As shown in Table 4, a high-cholesterol diet increases the serum HDL-C level of the $\mathrm{H}$ group by $53.9 \%(p<0.05)$, as compared to the $\mathrm{N}$ group. With regard to the effect on the LDL-C level, feeding a high-cholesterol diet to the $\mathrm{H}$ group resulted in a significantly higher LDL-C level than that of the $\mathrm{N}$ group $(p<$ $0.05)$. The LDL-C level significantly decreased in all test groups
Table 4. Effect of Milk-Soymilk and Fermented Milk-Soymilk on Serum HDL-C and LDL-C Levels in Hyperlipidemic Hamsters ${ }^{a}$

\begin{tabular}{llcc}
\hline group & HDL-C $(\mathrm{mg} / \mathrm{dL})$ & LDL-C $(\mathrm{mg} / \mathrm{dL})$ & LDL-C/HDL-C ratio \\
\hline $\mathrm{N}$ & $53.6 \pm 8.5 \mathrm{a}$ & $40.0 \pm 15.8 \mathrm{a}$ & $0.76 \pm 0.28 \mathrm{ab}$ \\
$\mathrm{H}$ & $82.5 \pm 18.4 \mathrm{~d}$ & $85.6 \pm 32.5 \mathrm{~b}$ & $1.13 \pm 0.25 \mathrm{~b}$ \\
$\mathrm{HP}$ & $66.8 \pm 13.6 \mathrm{abc}$ & $48.1 \pm 27.1 \mathrm{a}$ & $0.73 \pm 0.37 \mathrm{ab}$ \\
$\mathrm{HS}$ & $63.0 \pm 10.1 \mathrm{ab}$ & $49.6 \pm 23.3 \mathrm{a}$ & $0.87 \pm 0.36 \mathrm{ab}$ \\
HSF-101 & $66.8 \pm 8.7 \mathrm{abc}$ & $47.9 \pm 31.6 \mathrm{a}$ & $0.71 \pm 0.37 \mathrm{ab}$ \\
HSF-102 & $70.2 \pm 11.2 \mathrm{bcd}$ & $43.6 \pm 17.1 \mathrm{a}$ & $0.64 \pm 0.29 \mathrm{a}$ \\
HM & $67.8 \pm 11.6 \mathrm{bc}$ & $46.9 \pm 24.8 \mathrm{a}$ & $0.69 \pm 0.35 \mathrm{ab}$ \\
HMF-101 & $61.9 \pm 7.5 \mathrm{ab}$ & $51.1 \pm 27.4 \mathrm{a}$ & $0.87 \pm 0.36 \mathrm{ab}$ \\
HMF-102 & $77.1 \pm 15.9 \mathrm{~cd}$ & $43.8 \pm 12.4 \mathrm{a}$ & $0.57 \pm 0.13 \mathrm{a}$
\end{tabular}

\begin{abstract}
${ }^{a} \mathrm{~N}$, normal diet $(0 \%$ cholesterol); $\mathrm{H}$, high-cholesterol diet, $\mathrm{HP}$, probucol and high-cholesterol diet; HS, milk-soymilk and high-cholesterol diet; HSF-101, milk-soymilk fermented by L. paracasei subsp. paracasei NTU 101 and highcholesterol diet; HSF-102, milk-soymilk fermented by L. plantarum NTU 102 and high-cholesterol diet; HM, milk-soymilk supplement with M. charantia and highcholesterol diet; HMF-101, milk-soymilk supplement with M. charantia fermented by L. paracasei subsp. paracasei NTU 101 and high-cholesterol diet; HMF-102, milk-soymilk supplement with M. charantia fermented by L. plantarum NTU 102 and high-cholesterol diet. Data are presented as mean $\pm \mathrm{SD}(n=8)$, and numbers with a different letter were significantly different $(p<0.05)$ from each other.
\end{abstract}

Table 5. Effect of Milk-Soymilk and Fermented Milk-Soymilk on Blood SOD Activity, TAS Activity, and TBARS in Hyperlipidemic Hamsters ${ }^{a}$

\begin{tabular}{lcll}
\hline \multicolumn{1}{c}{ group } & SOD (units/mg of $\mathrm{Hb})^{b}$ & \multicolumn{1}{c}{${\text { TAS }(\mathrm{mmol} / \mathrm{L})^{c}}$} & ${\text { TBARS }(\mathrm{mM})^{d}}^{\mathrm{N}}$ \\
\hline $\mathrm{N}$ & $2.08 \pm 0.20 \mathrm{c}$ & $0.88 \pm 0.10 \mathrm{bcd}$ & $1.04 \pm 0.10 \mathrm{~b}$ \\
$\mathrm{H}$ & $1.44 \pm 0.13 \mathrm{a}$ & $0.71 \pm 0.11 \mathrm{ab}$ & $1.22 \pm 0.26 \mathrm{bc}$ \\
$\mathrm{HP}$ & $1.59 \pm 0.16 \mathrm{ab}$ & $0.87 \pm 0.09 \mathrm{bcd}$ & $1.12 \pm 0.17 \mathrm{bc}$ \\
$\mathrm{HS}$ & $1.43 \pm 0.23 \mathrm{a}$ & $0.66 \pm 0.21 \mathrm{a}$ & $1.19 \pm 0.18 \mathrm{bc}$ \\
$\mathrm{HSF}-101$ & $1.40 \pm 0.16 \mathrm{a}$ & $0.72 \pm 0.17 \mathrm{abc}$ & $0.70 \pm 0.10 \mathrm{a}$ \\
$\mathrm{HSF}-102$ & $1.63 \pm 0.19 \mathrm{ab}$ & $0.66 \pm 0.16 \mathrm{a}$ & $0.75 \pm 0.20 \mathrm{a}$ \\
HM & $1.91 \pm 0.24 \mathrm{c}$ & $0.93 \pm 0.21 \mathrm{~cd}$ & $1.29 \pm 0.27 \mathrm{c}$ \\
HMF-101 & $1.68 \pm 0.18 \mathrm{~b}$ & $0.99 \pm 0.20 \mathrm{~d}$ & $0.85 \pm 0.14 \mathrm{a}$ \\
HMF-102 & $1.89 \pm 0.13 \mathrm{c}$ & $0.89 \pm 0.17 \mathrm{bcd}$ & $0.82 \pm 0.11 \mathrm{a}$
\end{tabular}

${ }^{a} \mathrm{~N}$, normal diet $(0 \%$ cholesterol); $\mathrm{H}$, high-cholesterol diet, $\mathrm{HP}$, probucol and high cholesterol diet; HS, milk-soymilk and high-cholesterol diet; HSF-101, milk-soymilk fermented by L. paracasei subsp. paracasei NTU 101 and highcholesterol diet; HSF-102, milk-soymilk fermented by L. plantarum NTU 102 and high-cholesterol diet; HM, milk-soymilk supplement with M. charantia and highcholesterol diet; HMF-101, milk-soymilk supplement with M. charantia fermented by L. paracasei subsp. paracasei NTU 101 and high-cholesterol diet; HMF-102, milk-soymilk supplement with M. charantia fermented by L. plantarum NTU 102 and high-cholesterol diet. Data are presented as mean $\pm \operatorname{SD}(n=8)$, and numbers with a different letter were significantly different $(p<0.05)$ from each other. ${ }^{b}$ SOD $=$ superoxide dismutase ${ }^{c}$ TAS $=$ total antioxidant status. ${ }^{d}$ TBARS $=$ thiobarbituric acid reactive substances.

( $p<0.05$ ) when compared to that of the $\mathrm{H}$ group. According to the statistical comparison between test groups and the $\mathrm{H}$ group, milk-soymilk, fermented milk-soymilk, and normal diet groups have no significant difference on the effect of lowering the LDL-C level to each other. The results in Table 4 indicated that feeding a high-cholesterol diet for 8 weeks with fermented milk-soymilk by L. plantarum NTU 102 led to a decrease in the ratio of LDL-C/HDL-C, as compared to the $\mathrm{H}$ group ( $p<$ $0.05)$. Therefore, the results obtained by a statistical analysis shows that the ratio of LDL-C/HDL-C was less by $43.4 \%$ and $49.6 \%$ in the HSF-102 and HMF-102 groups than in the H group $(p<0.05)$. Fermented milk-soymilk and the supplement with $M$. charantia by L. plantarum NTU 102 were able to perform a significant effect $(p<0.05)$ on lowering the ratio of LDLC/HDL-C, but unfermented milk-soymilk was not significant $(p>0.05)$, as compared to the $\mathrm{H}$ group.

Anti-atherosclerotic Effect. As shown in Table 5, feeding the high-cholesterol diet for 8 weeks led to a decrease by $30.8 \%$ $(p<0.05)$ in SOD activity. Administration with unfermented 

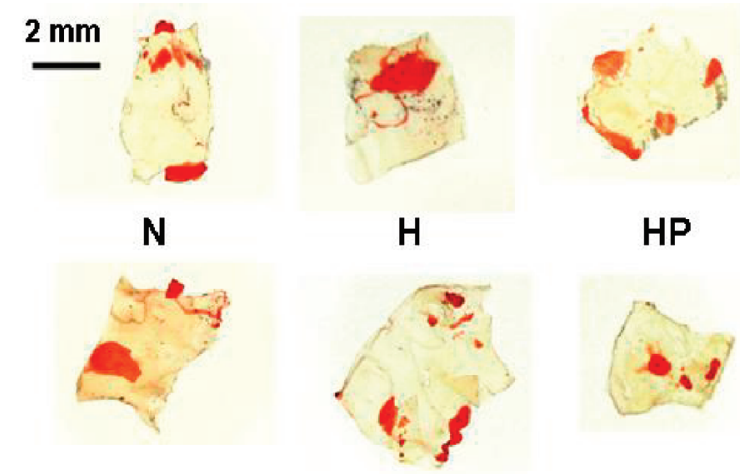

HP
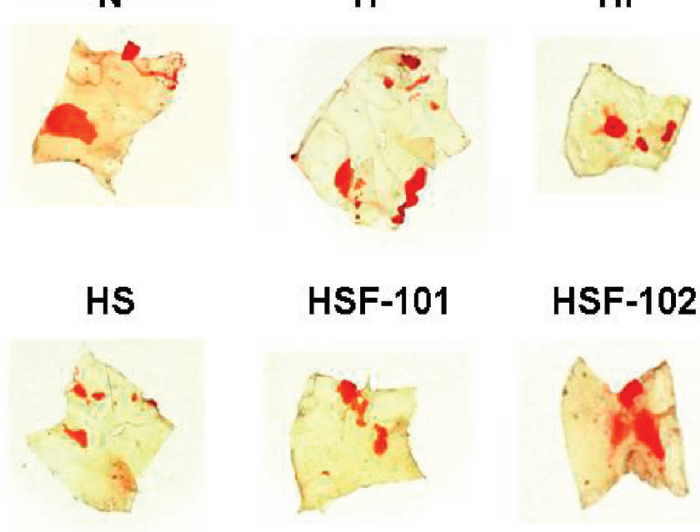

HSF-102

HM

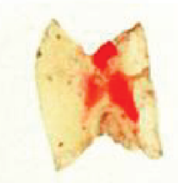

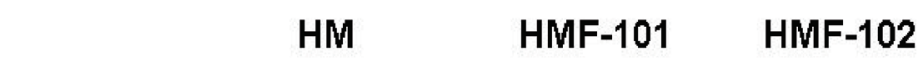

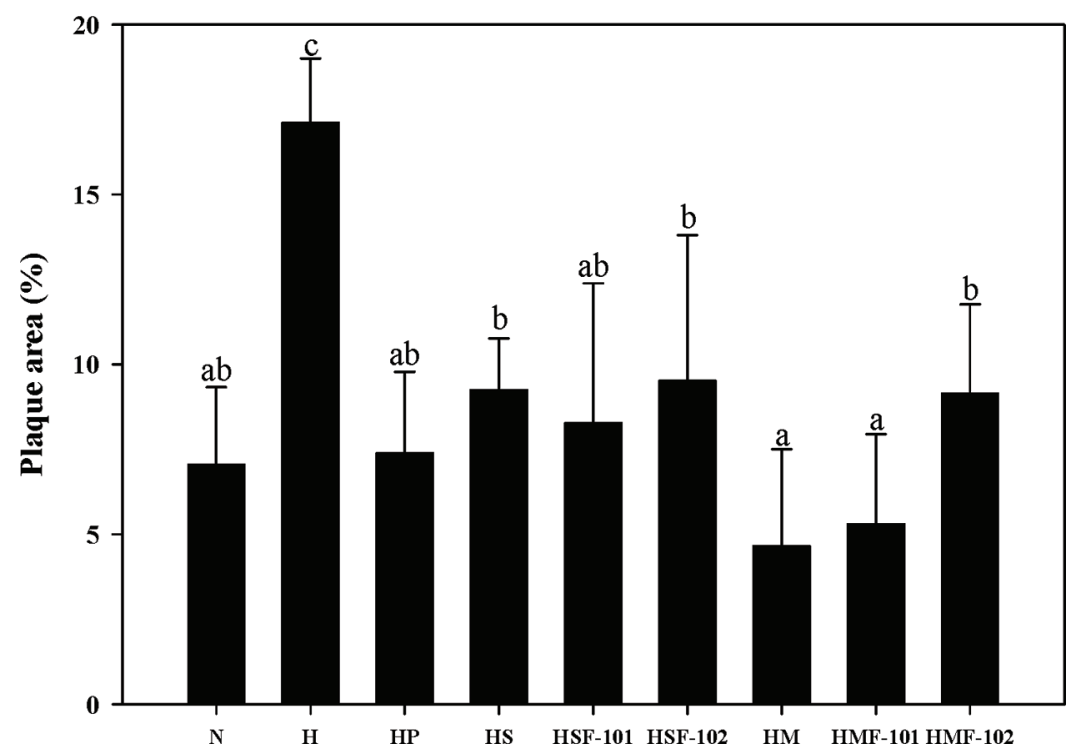

Figure 2. Effect of milk-soymilk and fermented milk-soymilk on atherosclerotic plaque in the thoracic aorta of hyperlipidemic hamsters. (Top) Atherosclerotic plaque presented as the red dye in the graph. (Bottom) Proportion of the area of atherosclerotic plaque in the aorta. The whole surface area of thoracic aorta was stained by Sudan IV and photographed using digital camera. The aortic surface area and its stained plaque area (red dye) were selected and quantitated by the Posterize program of Photoshop 7.0 software (Adobe Systems Incorporation, San Jose, CA). The selected pixel of plaque area and whole aorta was used to calculate the percent area of the aortic plaque. $\mathrm{N}$, normal diet ( $0 \%$ cholesterol); $\mathrm{H}$, high-cholesterol diet, $\mathrm{HP}$, probucol and high-cholesterol diet; HS, milk-soymilk and high-cholesterol diet; HSF-101, milk-soymilk fermented by L. paracasei subsp. paracasei NTU 101 and high-cholesterol diet; HSF-102, milk-soymilk fermented by L. plantarum NTU 102 and high-cholesterol diet; HM, milk-soymilk supplement with M. charantia and high-cholesterol diet; HMF-101, milk-soymilk supplement with M. charantia fermented by L. paracasei subsp. paracasei NTU 101 and high-cholesterol diet; HMF-102, milk-soymilk supplement with M. charantia fermented by L. plantarum NTU 102 and high-cholesterol diet. Data are presented as mean $\pm \mathrm{SD}(n=8)$, and mumbers with a different letter were significantly different $(p<0.05)$ from each other.

and fermented milk-soymilk supplemented with $M$. charantia and fermented by L. paracasei subsp. paracasei NTU 101 and L. plantarum NTU 102 significantly stimulated SOD activities by $32.6 \%(p<0.05), 16.7 \%(p<0.05)$, and $31.3 \%(p<0.05)$, respectively, but increased TAS by $31.0 \%(p<0.05)$ and $39.4 \%$ $(p<0.05)$ for HM and HMF-101, as compared to the H group.

TBARS assay was the general procedure for the evaluation of lipid peroxidation. In the results of Table 5, fermented milk-soymilk and fermented milk-soymilk supplement with $M$. charantia treatments significantly decreased the TBARS levels of serum in hamsters $(p<0.05)$, as compared to the $\mathrm{H}$ group. However, unfermented milk-soymilk groups did not have the ability to prevent the lipid peroxidation. The degree of TBARS was relieved by the fermented milk-soymilk treatment at a significant effect.

Atherosclerotic plaque in aorta caused by oxidative stress and lipid accumulation is the atheromatous lesions in atherosclerosis. As illustrated in Figure 2, the lipid plaque is largely accumulated at $142.5 \%$ in the aorta of hyperlipidemic hamsters. All test groups that included milk-soymilk and fermented milk-soymilk were significantly different from the $\mathrm{H}$ group $(p<0.05)$. The milk-soymilk supplement with $M$. charantia, HM and HMF- 
101 groups, resulted in a significant decrease by $72.8 \%$ and $69.0 \%$ in the percent area of the atheromatous lesions, as compared to the $\mathrm{H}$ group, respectively. Milk-soymilk and fermented milk-soymilk appear to significantly decrease the atherosclerotic plaque in aorta, which were compared to the $\mathrm{H}$ group.

\section{DISCUSSION}

Flavonoids are polyphenolic compounds found abundantly in plants, which may play a dietary role in reducing the risk of chronic diseases, such as cardiovascular disease and cancer, and it exists in nature almostexclusively as $\beta$-glycosidase enzymes $(20,21)$. These $\beta$-glycosidase enzymes were found in the brush border of the small intestine. Some microorganisms, such as Lactobacillus, were found to possess endogenous $\beta$-glucosidases (11), which play a role in the hydrolysis of isoflavone glycosides, such as Lactobacillus spp., Bacteroides spp., and Bifidobacterium spp. In humans, isoflavone aglycones were absorbed faster and in greater amounts than glucosides when ingested in the form of a beverage, such as soymilk (5). M. charantia is a common oriental vegetable in Taiwan, and some Taiwanese and Chinese literature described that $M$. charantia has many medical bioactivities. Previous studies have indicated that isoflavones exhibit free-radical-scavenging action, and soy is a rich source of antioxidants (22). Antioxidative stress is enhanced in the heart after myocardial infarction (23). A soy-enriched diet offered to rats could help to preserve their heart function after myocardial ischemia (24). On the other hand, $M$. charantia is able to reduce blood glucose and lipids in both normal and diabetic animals. It may protect $\beta$ cells, enhance insulin sensitivity, reduce oxidative stress, and possess immune-adjuvant activity (24). $M$. charantia also exhibited a marked reduction in the hepatic total cholesterol and triglyceride levels in both the presence and absence of dietary cholesterol (13). In this study, the activities of $\beta$-glucosidase by L. paracase $i$ subsp. paracase $i$ NTU 101 and L. plantarum NTU 102 and supplemented with $M$. charantia were also developed and investigated. As a result of hypocholesterol and 5-fold isoflavone aglycones, the developed milk-soymilk with or without $M$. charantia demonstrated that they were best suited as functional beverages.

Atherosclerosis constitutes the most common medical problem. This can be manifested clinically as stroke, coronary artery disease, or peripheral vascular disease (25). The causal relationship between the blood cholesterol level and atherosclerosis is widely accepted; there is already considerable interest in the possibility of using drugs to decrease cholesterol levels. In this study, milk-soymilk and fermented milk-soymilk were used to investigate the improvement of hyperlipidemic effects. The results demonstrated that all milk-soymilk and fermented milk-soymilk groups are proven to perform potential hypocholesterolemic effects, but there was no effect on the hypotriglyceridemic effect. Furthermore, the effect on HDL-C, LDL$\mathrm{C}$, and the ratio of LDL-C/HDL-C was significantly different on HMF-102 for both with and without M. charantia. The fermented group by L. plantarum NTU 102 for both with and without $M$. charantia had more potential than other groups, which were unfermented or fermented by L. paracasei subsp. paracasei NTU 101, to improve the ratio of LDL-C/HDL-C. Although all milk-soymilk groups, including unfermented and fermented ones, resulted in a hypocholesterolemic effect, the result can suggest that fermented milk-soymilk by $L$. plantarum NTU 102 with or without $M$. charantia had a greater hypocholesterolemic effect than others, as compared to the $\mathrm{H}$ group.

Oxidative stress is an important risk factor in the pathogenesis of atherosclerosis (26). The involvement of reactive oxygen species (ROS) in the oxidative modification of LDL, an important risk factor of atherogenesis, has drawn attention to the antioxidative defense of ROS scavengers in the organism. The antioxidative enzymes, so-called primary scavengers, in red blood cells and serum include SOD, seleno-dependent glutathione peroxidase, glutathione reductase, and catalase (27). The highly aggressive and toxic hydroxyl radicals have been proposed to play important pathogenic roles involved in attacking lipids, proteins, or DNA to acquire the missing electron. However, catalase conjugated to SOD represses the conversion of hydrogen peroxide to hydroxyl radicals. Superoxide anion radicals are dismuted by SOD to hydrogen peroxide and oxygen. Under physiological conditions, the conjugation of catalase to SOD ensures that, as soon as a superoxide dismutation reaction occurs, the resultant $\mathrm{H}_{2} \mathrm{O}_{2}$ is removed by the immediate proximity of the catalase molecule (27). A high LDL-C level certainly leads to both a decreased activity of antioxidative mechanisms and an excess of substrate, which can undergo abnormal oxidative modifications (26). In the current study, the increase of TAS and SOD activity and the repression of lipid oxidation in blood of hyperlipidemic hamsters were exhibited by the milk-soymilk supplemented with $M$. charantia and fermented by L. paracase $i$ subsp. paracase $i$ NTU 101. The product of lipid peroxidation in blood was also determined by the assay of TBARS formation, and the formation of TBARS in blood of hyperlipidemic hamsters was inactivated in fermented groups by L. paracasei subsp. paracasei NTU 101 and L. plantarum NTU 102. The formation of an atherosclerotic plaque is proven to be increased by increasing the level of oxLDL and oxidative stress of the blood circulation. The formations of atherosclerotic plaque were repressed by milk-soymilk with or without fermentation and supplemented with $M$. charantia. In conclusion, fermented milk-soymilk by L. paracase $i$ subsp. paracasei NTU 101 with $M$. charantia was able to resist hyperlipidemia-induced oxidative stress.

Thus, the major principle outcome of this study is to demonstrate, for the first time, an improvement in the hypolipidemic and anti-atherosclerotic activity with the milk-soymilk, milk-soymilk supplement with $M$. charantia, and fermented milk-soymilk by L. paracase $i$ subsp. paracasei NTU 101 and L. plantarum NTU 102 isolated from Taiwan. The overall results confirm the preventive roles of soy-derived products supplemented with $M$. charantia by fermenting with $L$. paracase $i$ subsp. paracasei NTU 101. Our results indicate that these beneficial effects are significant hypocholesterolemic effects and potentially antioxidative to prevent and retard hyperlipidemiainduced oxidative stress and atherosclerosis.

\section{LITERATURE CITED}

(1) Liu, J. R.; Lin, C. W. Production of kefir from soymilk with or without added glucose, lactose, or sucrose. J. Food Sci. 2000, 65, 716-719.

(2) Bannwart, C.; Fotsis, T.; Heikkinen, R.; Adlercreutz, H. Identification of the isoflavonic phytoestrogen daidzein in humanurine. Clin. Chim. Acta 1984, 136, 165-172.

(3) Choi, I.; Kim, Y.; Park, Y.; Seog, H.; Choi, H. Anti-obesity activities of fermented soygerm isoflavones by Bifidobacterium breve. Biofactors 2007, 29, 105-112.

(4) Nielsen, I. L.; Williamson, G. Review of the factors affecting bioavailability of soy isoflavones in humans. Nutr. Cancer 2007, 57, 1-10.

(5) Kano, M.; Takayanagi, T.; Harada, K.; Sawada, S.; Ishikawa, F. Bioavailability of isoflavones after ingestion of soy beverages in healthy adults. J. Nutr. 2006, 136, 2291-2296. 
(6) Chun, J.; Kim, G. M.; Lee, K. W.; Choi, I. D.; Kwon, G. H.; Park, J. Y.; Jeong, S. J.; Kim, J. S.; Kim, J. H. Conversion of isoflavone glucosides to aglycones in soymilk by fermentation with lactic acid bacteria. J. Food Sci. 2007, 72, M39-M44.

(7) Holzapfel, W. H.; Hhaberer, P.; Geisen, R.; Bjorkroth, J.; Schillinger, U. Taxonomy and important features of probiotic microorganisms in food and nutrition. Am. J. Clin. Nutr. 2001, 73, 365-373.

(8) Tsai, Y. T.; Cheng, P. C.; Fan, C. K.; Pan, T. M. Time-dependent persistence of enhanced immune response by a potential probiotic strain Lactobacillus paracasei subsp. paracasei NTU 101. Int. J. Food Microbiol. 2008, 128, 219-225.

(9) Sit, C. S.; Vederas, J. C. Approaches to the discovery of new antibacterial agents based on bacteriocins. Biochem. Cell Biol. 2008, 86, 116-123.

(10) Wells, J. M.; Mercenier, A. Mucosal delivery of therapeutic and prophylactic molecules using lactic acid bacteria. Nat. Rev. Microbiol. 2008, 6, 349-362.

(11) Otieno, D. O.; Shah, N. P. Endogenous $\beta$-glucosidase and $\beta$-galactosidase activities from selected probiotic micro-organisms and their role in isoflavone biotransformation in soymilk. J. Appl. Microbiol. 2007, 103, 910-917.

(12) Huang, L.; Ikejiri, A.; Shimizu, Y.; Adachi, T.; Goto, Y.; Toyama, J.; Tanaka, H.; Akashi, R.; Uchida, K.; Miyata, H.; Haga, T. Immunoadjuvant activity of crude lectin extracted from $M$. charantia seed. J. Vet. Med. Sci. 2008, 70, 533-535.

(13) Yin, J.; Zhang, H.; Ye, J. Traditional chinese medicine in treatment of metabolic syndrome. Endocr., Metab. Immune Disord.: Drug Targets 2008, 8, 99-111.

(14) Chiu, C. H.; Lu, T. Y.; Tseng, Y. Y.; Pan, T. M. The effects of Lactobacillus-fermented milk on lipid metabolism in hamsters fed on high-cholesterol diet. Appl. Microbiol. Biotechnol. 2006, 71, $238-245$

(15) Lin, S. J.; Lay, H. L.; Wu, S. T.; Thseng, F. S. Contents of certain isoflavones in Glycine dolichocarpa, G. tabacina and G. tomentella collected in Taiwan. J. Food Drug Anal. 2005, 13, 260266.

(16) Boyd, E. The Growth of the Surface Area of Human Body; University of Minnesota Press: Minneapolis, MN, 1935.

(17) Chen, J. R.; Liu, S. M.; Yang, S. C.; Suetsuna, K. Soymilk intake is associated with plasma and liver lipid profiles in rats fed a highcholesterol diet. Nutrition 2004, 20, 929-933.
(18) Mabile, L.; Fitoussi, G.; Periquet, B.; Schmitt, A.; Salvayre, R.; Negre-Salvayre, A. $\alpha$-Tocopherol and trolox block the early intracellular events (TBARS and calcium rises) elicited by oxidized low density lipoproteins in cultured endothelial cells. Free Radical Biol. Med. 1995, 19, 177-187.

(19) Miller, N. J.; Rice-Evans, C.; Davies, M. J.; Gopinathan, V.; Milner, A. A novel method for measuring antioxidant capacity and its application to monitoring the antioxidant status in premature neonates. Clin. Sci. 1993, 84, 407-412.

(20) Middleton, E.; Kandaswami, C. The impact of plant flavonoids on mammalian biology: Implications for immunity, inflammation and cancer. In The Flavonoids Advances in Research since 1986; Harborne, J. B., Ed.; Chapman and Hall: London, U.K., 1993; pp 619-652.

(21) Salah, N.; Miller, N. J.; Paganga, G.; Tijburg, L.; Bolwell, G. P.; Rice-Evans, C. Polyphenolic flavanols as scavengers of aqueous phase radicals and as chain-breaking antioxidants. Arch. Biochem. Biophys. 1995, 322, 339-346.

(22) Rufer, C. E.; Kulling, S. E. Antioxidant activity of isoflavones and their major metabolites using different in vitro assays. J. Agric. Food Chem. 2006, 54, 2926-2931.

(23) Hill, M. F.; Singal, P. K. Antioxidant and oxidative stress changes during heart failure subsequent to myocardial infarction in rats. Am. J. Pathol. 1996, 148, 291-300.

(24) Hagen, M. K.; Lehenbauer-Lüdke, A. R.; Paludo, A. C.; Schenkel, P.; Gonçalves, L.; Fernandes, T. G.; Caron, R.; Llesuy, S.; Mill, J. G.; Belló-Klein, A. Diet with isolated soy protein reduces oxidative stress and preserves ventricular function in rats with myocardial infarction. Nutr. Metab. Cardiovasc. Dis. 2009, in press.

(25) Kratz, M. Dietary cholesterol, atherosclerosis and coronary heart disease. Handb. Exp. Pharmacol. 2005, 170, 195-213.

(26) Jessup, W.; Dean, R. T.; de Whalley, C. V.; Rankin, S. M.; Leake, D. S. The role of oxidative modification and antioxidants in LDL metabolism and atherosclerosis. Adv. Exp. Med. Biol. 1990, 264, 139-142.

(27) Liczmanski, A. E. Oxygen toxicity. II. Defense mechanisms. Postepy Biochem. 1988, 34, 293-310.

Received for review September 19, 2008. Revised manuscript received January 18, 2009. Accepted January 19, 2009.

JF802936C 